
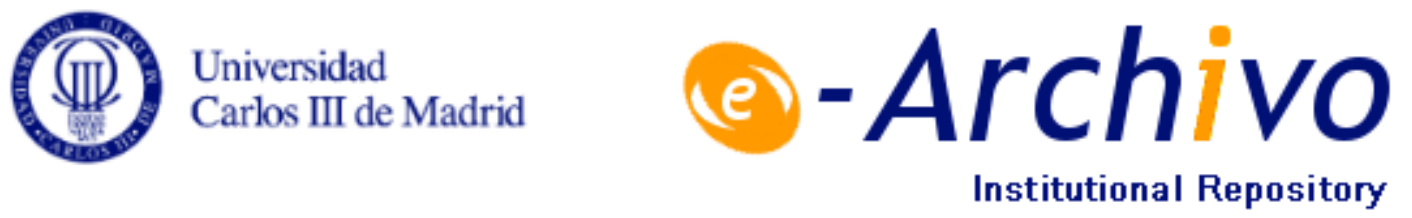

This is a postprint version of the following published document:

Beviá, C., Corchón, Luis C. and Romero-Medina, A. (2016). Relinquishing power, exploitation and political unemployment in democratic organizations. Social Choice and Welfare, pp. $1-19$.

Available in: https://doi.org/10.1007/s00355-016-0989-5

(C) Springer Verlag 


\title{
Relinquishing power, exploitation and political unemployment in democratic organizations
}

\author{
Carmen Beviá ${ }^{1}$. Luis Corchón ${ }^{2}$. \\ Antonio Romero-Medina ${ }^{2}$
}

\begin{abstract}
We analyze the evolution of organizations which take decisions on whom to hire and how to share the output by plurality voting. Agents are grouped in three classes, high, medium and low productivity. We study the evolution of political power and show that in some cases, rational agents who value the future may yield political power to another class. This is what we call the relinquish effect. We show that high productivity agents may receive less than their individual output, i.e. exploitation is possible. We also show that high productivity agents may be left out in the cold because their entrance in an organization may threaten the dominance of other classes. We call this political unemployment.
\end{abstract}

\section{Introduction}

The last two decades have seen a growing interest in the dynamic side of organizations. In particular, organizations evolve over time, changing in size, internal composition

\footnotetext{
Thanks to Humberto Llavador, Jan Zapal, James Schummer, two referees and participants in seminars in which this paper was presented for very helpful comments. Thanks to the MOMA network under the Project ECO2014-57673-REDT for financial support. The first author acknowledges financial support from ECO2014 53051, SGR2014-515 and PROMETEO/2013/037. Luis Corchon and Antonio Romero-Medina acknowledge financial support from MEC under Project ECO2014_57442_P, and financial support from the Ministerio Economía y Competitividad (Spain), Grants MYGRANT and MDM 2014-0431. Luis Corchon acknowledges the hospitality of the departments of economics at Cambridge (UK) and MEDS, Northwestern U. during the writing of this paper. Antonio Romero-Medina acknowledges the hospitality of the department of economics at Boston College.
}

$\triangle$ Carmen Beviá

carmen.bevia@gmail.com

1 Universidad de Alicante, Alicante, Spain

2 Universidad Carlos III de Madrid, Getafe, Spain 
and distribution of output. The example that comes to mind are university departments, but our main ideas can be applied to other settings like cooperative firms or political representation.

As a starting point, one may think that when the future is not an important factor, a (rational) dominant class may take actions that will endanger the political supremacy of this class in the future. This corresponds to the dictum by French king Louis XV "Apres moi le deluge". But when the future plays a decisive role, one would not expect a (rational) dominant political class to take actions that endanger their political supremacy. We may call this conjecture "The Iron Law of Political Power". According to this law, a dominant class will peacefully endorse the political supremacy to another class only if (a) it is useful in the fight against a common enemy and agents discount heavily the future (Llavador and Oxoby 2005), (b) it provides a Pareto improvement (Lizzeri and Persico 2004) or (c) when the threat of violent deposition is credible (Acemoglu and Robinson 2000; Conley and Temini 2001) In this paper, we present a three-class model in which this law does not hold.

We consider an organization with a duration of an infinite number of periods. Agents outside the organization are called outsiders and those inside the organization are called insiders. These agents are distinguished by their productivity and can be of three types: $H$ (high), $M$ (medium) or $L$ (low). We will refer to each type as a class. There is a pool of outsiders of all types who are potential entrants in the organization. In each period, the organization takes three decisions sequentially. First, insiders decide by majority voting how to share the output produced in this period. Second, insiders decide by plurality who joins the organization. For simplicity, we will assume that the organization hires just one new member every period. The hired agent will participate in the voting of this rule and in the hiring of a new member from the next period on. Next, the agents who wish, leave the organization and join the pool of agents outside the organization. The leaving agents receive their reservation utility outside the organization. Finally, production takes place and output is shared among insiders, according to the rule that the organization has voted. A class has the political power -or is dominant- when a member of this class is a pivotal voter in the voting on the sharing rule.

We show that if $H$ is the dominant class at time 0 , they will vote meritocracy and they will hire $H$ types only. In any other case, if the discount factor $\delta$ is close to one and the organization is capable of producing a surplus, $M$ type agents become pivotal and, eventually, they will vote for egalitarianism and they will hire $M$ and $H$ agents in such a way that they stay pivotal. In particular, if $L$ agents are the dominant class at the starting time, they will relinquish power to the $M$ class. This is because, in the long run, the interest of the middle and lower classes regarding distribution are aligned: they prefer an egalitarian distribution scheme. In addition, hiring members of the middle class strengthens production and with an egalitarian policy this improves the payoffs of the lower class. We call this the Relinquish effect. This effect happens any time that a dominant class relinquishes power to another class that may be more efficient managing the economy. This class will not alter either the rules or the outcome of the game. The rules may be voting or not. For example, in imperial China under the Tang dynasty, the oligarchy relinquished a lot of their power to the Mandarin class, which was more efficient than the feudal lords, to extract surplus from the farmers and had no 
intention of subverting the social order. Our paper contributes to the understanding of how power is maintained (or lost) in organizations and how it affects the distribution of resources.

In our model, initial insiders determine the long term performance of the organization. In particular, organizations in which the high type is not dominant in the first period, will never achieve a majority of high type agents, even though the percentage of high types can be close to fifty percent. All high type agents have to share their output with the other types, so they are paid less than their productivity. We say that these agents are Exploited. And since there are high type agents that could have been hired, we say that we have Political Unemployment, i.e. unemployment due to the political constraints inside the organization (in this case, plurality voting).

Next, we consider finitely living agents. In this scenario we see that the demographic structure at time zero plays an important role. In some cases, there is convergence to the equilibrium where meritocracy holds even if we start with a majority of low types.

Our paper focuses on the policies followed by rational classes and is written in the spirit of the monumental "A General Theory of Exploitation and Class" (1982a) in which John Roemer laid the foundations of a rigorous analysis of exploitation and class. In this book and several posterior writings (1982b, 1985, 1988) John Roemer uses the methods of Walrasian general equilibrium and game theory to show how exploitation and class relations arise from differential ownership of productive assets. Our methods are different -voting and dynamic game theory barely existed then. Therefore, our results are not directly comparable. Our framework does not allow us to differentiate between different classes based on the property of assets such as labor or capital. We only have different skills. However, our model, as in Roemer (1982a) Chapter 2, verifies “ a key Marxian insight - that membership in a laborhiring class is associated with exploiting, and membership in a labor selling class is associated with being exploited" (p. 15, 1. 6.). Roemer's specification also finds that "exploitation is also viable when there are differential skills" (p. 20, 1. -7) which is compatible with our findings. Finally, Roemer mentions the possibility of what he calls status exploitation. This phenomenon is similar to the one that arises under the ruling class in our organization because our model forces agents who join the organization to produce. In his words: " status, exploitation ... is the inequality which arises as a consequence of unequal access to privileged positions." (p. 22, 1. 4).

Our paper contributes to the dynamic theory of democratic organizations and so it covers similar grounds to papers studying elections in stationary environments (see Duggan and Martinelli 2015, for a survey), models of legislative bargaining (see Dziuda and Loeper 2015; Zapal 2014) and models of endogenous constituency (see Roberts 2015; Razin et al. 2016). The first paper with an endogenous set of voters is by Barberá et al. (2001). They showed that equilibrium may follow a complicated pattern like agents voting against their short term interest in order to enhance their long term payoff. The closest paper is by Jack and Lagunoff (2006). They show that an enfranchised elite may voluntarily dilute its power by expanding the franchise because this allows the current pivotal voter to credibly commit to future policy choices. In equilibrium, expansion occurs if and only if the private decisions of the citizenry have a net positive spillover to the dynamic payoff of the current pivotal voter. This is similar to our model in which a class relinquishes power because it is in its interest. Their 
model, though, is very different from ours: technically, because it uses differential calculus and in our model decisions are taken on integers. Also in their model, there is no concern for topics that are central here like exploitation or political unemployment. Finally, a recent paper by Barberá et al. (2015) also tackles the issues of egalitarianism and meritocracy using a model of coalition formation. Decisions are taken by majority voting inside each coalition. As in our model, the inability of voters to commit to a distributional rule is a potential source of trouble.

The remainder of the paper is organized as follows. Section 2 introduces the basic model. Section 3 presents our main results. Section 4 extends the basic model to finitely-living agents. Section 5 concludes.

\section{The model}

An organization is a productive facility. Some agents belong to the organization and are called insiders, and some do not belong to the organization and are called outsiders. Both the organization and all agents last for a countable infinite number of periods $\tau=(0,1, \ldots t, \ldots)$.

Agents can be of three types: $H, M$ and $L$. If an agent of type $T \in\{H, M, L\}$ is an insider, she produces a per period gross output of $o_{T}$. The per period reservation utility of an agent of type $T \in\{H, M, L\}$ is denoted by $u_{T}$. Let $x_{T} \equiv o_{T}-u_{T}$ be the surplus (or net output) that the organization obtains from an agent of type $T$. Assume that $x_{H}>x_{M}>x_{L}>0$. Thus type $H$ is the high productivity type, $M$ is the medium productivity type, and $L$ is the low productivity type. Given that surplus is positive we can assume without loss of generality that $u_{T}=0$.

In each period $\tau$, the organization takes two decisions in turn. First, the insiders in period $\tau-1$ decide how the total surplus in $\tau$ is going to be shared. Second, they decide who joins the organization and the outsiders decide if they accept the offer or not. Next, the agents who wish, leave the organization and receive their reservation utility. Finally, production takes place and output is shared among the new insiders. The timing of the decisions is motivated by the fact that the decision on the distributive rule must be made in advance of the next hire, if not, insiders would not know the distributive effects of the hiring and outsiders would not know the exact offer made to them. Furthermore, if agents leave the organization before voting, this would be equivalent to considering an organization with a different set of agents. Thus, changes in population due to exit will have its effect both at the production moment and in the next period decision stage.

If there are $n_{T}^{\tau}$ insiders of type $T=H, M, L$ in period $\tau$ (including the new hiring in this period), the surplus to be shared, denoted by $X^{\tau}$, is $X^{\tau} \equiv n_{H}^{\tau} x_{H}+n_{M}^{\tau} x_{M}+n_{L}^{\tau} x$. All insiders in period $\tau$ are paid a share in the surplus denoted by $c_{T}^{\tau} / X^{\tau}$. We refer to $c_{T}^{\tau}$ as the consumption of an agent of type $T$ at period $\tau$. An agent's payoff at $\tau$ is just her consumption. All agents have a common discount factor $\delta<1$. An agent's intertemporal payoff at $\tau$ is given by $\sum_{t=0}^{\infty} \delta^{t} c_{T}^{\tau+t}$.

At the beginning of period 0 , the organization is populated by some insiders and has a rule (i.e. a constitution) that fixes how collective decisions are taken. In this 
paper, we concentrate on the case in which decisions on hiring and on the distribution of surplus are taken by plurality.

In order to simplify the model we make the following assumptions.

Assumption A1. The pool of outsiders includes agents from the three types at the start of the game and at any history of the game.

Assumption A2. The organization hires a single outsider per period.

Assumption A3. Output is distributed by the following sharing rule

$$
\frac{c_{T}^{\tau}}{X^{\tau}}=\theta^{\tau} \frac{1}{n_{H}^{\tau}+n_{M}^{\tau}+n_{L}^{\tau}}+\left(1-\theta^{\tau}\right) \frac{x_{T}}{X^{\tau}},
$$

where $c_{T}^{\tau}$ is the output allocated to an agent of type $T$ in the period $\tau$ and $\theta^{\tau} \in[0,1]$.

Assumption A4. Whenever there is a tie, the highest productive agent in the organization decides. If an agent is indifferent between hiring agents from different types, she will vote for the agent from the highest type.

Assumption A5. Agents vote sincerely.

Several comments are in order: A1 is made to discard problems associated with a rigid labor supply.

A2 is made to simplify the voting. The choice of the sharing rule described in A3 is motivated by a result by Kang (1988) showing that for $n>2$ it is the unique differentiable sharing rule which is symmetric and homogeneous of degree zero. The last property is a desirable one because it guarantees that the share does not depend on the units in which it is measured. The deterministic tie-breaking rule in A4 is chosen to avoid lotteries that might complicate the history of the game. Our tie breaking rules favour excellency. Despite this, we will see that in equilibrium excellency is not always achieved. A5 is made in order to destroy equilibria in which all agents vote for the alternative which is at the bottom of their preferences. It means that each agent believes that her vote is pivotal. Without this assumption we may have very complicated vote patterns (that are highlighted in the paper by Barberá et al. 2001) and a serious problem of multiplicity of equilibria. This assumption can be understood as a refinement of equilibria. $^{1}$

Since the emphasis of our paper is on class interests, we assume that each member of each class (types) behaves as if she were in command of her class.

At the beginning of each period $\tau$, the history of the game can be summarized by a state, $s^{\tau}$, assumed to be the number of insiders at $\tau-1$, that is, the voters in period $\tau$. Thus, $s^{\tau}=\left(n_{H}^{\tau-1}, n_{M}^{\tau-1}, n_{L}^{\tau-1}\right)$. The state at $\tau+1$ depends on $s^{\tau}$ and the action taken at $\tau$. Let $S$ be the set of all possible states. We only consider strategies that are state dependent. By our assumption on sincere voting, the strategies for all members of a class can be summarized in a strategy for a typical member of this class. Let

\footnotetext{
1 Mavridis and Serena (2015) offered an appealing solution to the problem of multiplicity of Nash equilibrium in voting games when voting is costly.
} 
$\sigma_{T}: S \rightarrow\{0,1\} \times\{M, L, H\}$ be a strategy for a member of type $T \in\{L, M, H\}$. Thus a strategy for a typical member of a type is a mapping that yields the vote of this person on the sharing rule and on the hiring for each state. Given that the total net surplus is always positive, no insider wants to leave and no outsider will ever reject an offer to join the organization. This is why we do not model the strategy of an outsider and we do not consider the exit option.

Our equilibrium concept is Markov perfect equilibrium (MPE in what follows). We focus on Markovian equilibrium (as the papers quoted in the introduction did) for several reasons: they constitute the simplest sort of equilibrium and are thus perhaps focal (Duffie et al. 1994). And they provide a simple way to model some kind of coordination "an equilibrium which does not display minimal regularity through time -maybe stationarity- is unlikely to generate the coordination between agents that it assumes" (Guesnerie and Woodford 1992, Section 3).

Finally, note that since the sharing rule is decided in each period, when voting on it, agents only take into account the consumption in this period. Agents of type $L$ (resp. $H$ ) would always prefer $\theta^{\tau}=1$ (resp. $\theta^{\tau}=0$ ). The preferences of agents of type $M$ will depend on the group structure of the organization. If $x_{M} \leq X^{\tau} /\left(n_{H}^{\tau}+n_{M}^{\tau}+n_{L}^{\tau}\right)$, they will prefer $\theta^{\tau}=1$, and if $x_{M}>X^{\tau} /\left(n_{H}^{\tau}+n_{M}^{\tau}+n_{L}^{\tau}\right)$ they will prefer $\theta^{\tau}=0$. Hence, without loss of generality, we assume that voting takes place between $\theta^{\tau}=0$ -a meritocratic rule- and $\theta^{\tau}=1$-an egalitarian rule.

\section{Results}

We analyze the influence of the ruling class on the evolution of the organization in terms of both political power and resource distribution.

We say that a type $T \in\{H, M, L\}$ is the dominant class at time $\tau$ if $n_{T}^{\tau-1}>$ $n_{J}^{\tau-1}+n_{K}^{\tau-1}, J, K \in\{H, M, L\} \backslash\{T\}$. If $T$ is the dominant class, the pivotal voter is an agent of type $T$.

Our main result is the following:

Proposition 1 There exist $\bar{\delta}>0$ such that for all $\delta \geq \bar{\delta}, T \in\{H, M, L\}$ then:

(i) In any MPE if $H$ is the dominant class in period $\tau$, then $H$ is dominant in all subsequent periods and meritocracy will be the sharing rule.

(ii) In any MPE if $M$ is the pivotal type in period $\tau$, then $M$ will be the pivotal type in any subsequent period and eventually, egalitarianism will be the sharing rule.

(iii) In any MPE if $L$ is the dominant class in period $\tau$, then eventually egalitarianism will be the sharing rule, although they will eventually lose political power in favor of the medium class.

We start by proving the first part of the proposition. For the other parts we need a series of auxiliary results that we present here. The proofs of these auxiliary results are given in the Appendix.

Proof of $(i)$ A type $H$ agent will always vote for a meritocratic sharing rule in any possible state. Furthermore, given A4, she will vote for hiring a high type agent. Thus, if $H$ is the dominant class in $\tau$, they will be the dominant class in any subsequent period. 
Our first auxiliary result points out that, when there is no dominant class, for a sufficiently high $\delta$, the pivotal voter is a medium type. When deciding on the sharing rule, a high type agent will always vote for a meritocratic sharing rule and a low type agent for an egalitarian rule independently on the hiring decision. Thus, when there is no dominant class the pivotal voter is a medium type agent. The following lemma provides conditions under which, with no dominant class, the medium type is also pivotal in the hiring decision.

Lemma 1 There exist $\bar{\delta}_{0}>0.5$ such that for all $\delta \geq \bar{\delta}_{0}$ in any MPE, if in period $\tau$ there is no a dominant class, a type $M$ agent is the pivotal voter.

Lemma 1 formalizes the idea that the middle class enjoys a strategic advantage with respect to both "ends", low and high. And by exploiting the fact that one of the extremes will vote with the middle class, this class is pivotal in the voting game when the future is very important. And, again, when the future is very important, the middle class will target its votes on hiring to maintain this pivotal position in the long run.

In the following lemma we show that whenever $X^{\tau-1} / n^{\tau-1}<x_{M}<\left(X^{\tau-1}+\right.$ $\left.x_{H}\right) / n^{\tau}$, there exist $\bar{\delta}_{1}$ such that for both, medium and low type, having meritocracy today and egalitarianism from tomorrow onwards and alternating the hiring between a medium type and a high type starting by a medium type, is better than having egalitarianism today and hiring a high type and meritocracy from tomorrow onwards. Let $V_{E}^{1}(\delta)$ be the continuation payoff in the first case.

Lemma 2 Let $X^{\tau-1} / n^{\tau-1}<x_{M}<\left(X^{\tau-1}+x_{H}\right) / n^{\tau}$. There exist $\bar{\delta}_{1}>0.5$ such that for all $\delta>\bar{\delta}_{1}$,

$$
\begin{aligned}
& x_{M}+\delta V_{E}^{1}(\delta)>\left(X^{\tau-1}+x_{H}\right) / n^{\tau}+\frac{\delta}{1-\delta} x_{M}, \quad \text { and } \\
& x_{L}+\delta V_{E}^{1}(\delta)>\left(X^{\tau-1}+x_{H}\right) / n^{\tau}+\frac{\delta}{1-\delta} x_{L} .
\end{aligned}
$$

In the following Lemma we show that whenever $x_{M}<X^{\tau-1} / n^{\tau-1}<\left(X^{\tau-1}+\right.$ $\left.x_{H}\right) / n^{\tau}$, there exist $\bar{\delta}_{2}$ such that for both, medium and low type, having egalitarianism from now on and alternating the hiring between a medium type and a high type starting by a medium type, is better than egalitarianism today and hiring a high type and meritocracy from tomorrow onwards. Let $V_{E}^{2}(\delta)$ be the continuation payoff in the first case.

Lemma 3 Let $x_{M}<X^{\tau-1} / n^{\tau-1}<\left(X^{\tau-1}+x_{H}\right) / n^{\tau}$. There exist $\bar{\delta}_{2}$ such that for all $\delta>\bar{\delta}_{2}$,

$$
\begin{aligned}
& \left(X^{\tau-1}+x_{M}\right) / n^{\tau}+\delta V_{E}^{2}(\delta)>\left(X^{\tau-1}+x_{H}\right) / n^{\tau}+\frac{\delta}{1-\delta} x_{M}, \quad \text { and } \\
& \left(X^{\tau-1}+x_{M}\right) / n^{\tau}+\delta V_{E}^{2}(\delta)>\left(X^{\tau-1}+x_{H}\right) / n^{\tau}+\frac{\delta}{1-\delta} x_{L}
\end{aligned}
$$

We are now prepared to prove our main result. 
Proof of Proposition 1 (ii) First of all, note that in all $\tau$ such that $n_{H}^{\tau-1}+1<n_{M}^{\tau-1}+$ $n_{L}^{\tau-1}$, a medium type will always prefer to hire a high type because its pivotal position on the sharing rule for the next period is not at risk. The interesting periods are the ones in $n_{H}^{\tau-1}+1=n_{M}^{\tau-1}+n_{L}^{\tau-1}$. In those periods, by voting for a high type, the medium class will lose its pivotal position from there on. However, let us see that for a sufficiently high $\delta$, the medium type will prefer to keep the power. Since the decision on the sharing rule can be reversed in the next period, only today's payoffs are relevant when voting for different rules. We distinguish three cases.

Case ii1. Suppose that $x_{M} \leq X^{\tau-1} / n^{\tau-1}<\left(X^{\tau-1}+x_{H}\right) / n^{\tau}$.

In this case the medium type will vote for egalitarianism independently of the hiring. But by voting for a high type, the medium class will have egalitarianism today and meritocracy forever because high types will become the dominant class. However, if they vote for a medium type they will keep the power in the next period where again, there is no risk of losing power and a high type will be hired. In this case, they will have egalitarianism forever. By Lemma 3 there exist $\bar{\delta}_{2}>0$ such that for all $\delta \geq \bar{\delta}_{2}$, alternating the hiring between medium and high types is better than hiring a high type.

Case ii2. Suppose that $\left(X^{\tau-1}+x_{H}\right) / n^{\tau} \leq x_{M}$.

In this case the medium type will vote for a meritocratic rule independently of the hiring. But by voting for a high type, the medium class will have meritocracy forever because high types will become the dominant class. However, if they vote for a medium type they will keep the power in the next period where again, there is no risk of losing power and a high type will be hired. From period $\tau$ it is better to alternate the hiring between medium and high starting by hiring a medium type. This will be profitable compared with hiring a high type because eventually, in some period $\tau^{\prime}, x_{M}<\left(X^{\tau-1}+x_{H}\right) / n^{\tau}$, and the medium type, by keeping the power, will get egalitarianism from there on. Case iil shows that this is better than obtaining meritocracy forever.

Case ii3. Suppose that $X^{\tau-1} / n^{\tau-1} \leq x_{M}<\left(X^{\tau-1}+x_{H}\right) / n^{\tau}$.

First of all, note that if the medium voter is pivotal in this case, it is because $\delta \geq \bar{\delta}_{0}$ (by Lemma 1). But by voting for a high type, the medium class will have egalitarianism today and meritocracy forever because high types will become the dominant class. However, if they vote for a medium type they will keep the power in the next period where again, there is no risk of losing power and a high type will be hired. In this last case, they will have meritocracy today, but egalitarianism from tomorrow on. By Lemma 2 there exist $\bar{\delta}_{1}$ such that for all $\delta \geq \bar{\delta}_{1}$, keeping power is the best option.

Let $\bar{\delta}_{M}=\max \left\{\bar{\delta}_{0}, \bar{\delta}_{1}, \bar{\delta}_{2}\right\}$, by cases ii1, ii2, and ii3, for all $\delta \geq \bar{\delta}_{M}$, if $M$ is the pivotal type at period $\tau$, then $M$ will be the pivotal type in any subsequent period and eventually, egalitarianism will be the sharing rule.

(iii) Type $L$ will always vote for an egalitarian rule. Furthermore, whenever $n_{L}^{\tau-1}>$ $n_{M}^{\tau-1}+n_{H}^{\tau-1}+1$ they will vote for a high type and they will keep power in the next period. The interesting periods are the ones in which $n_{L}^{\tau-1}=n_{M}^{\tau-1}+n_{H}^{\tau-1}+1$. In those periods, by voting for a high type the lower class will cease to be the dominant class. If $n_{M}^{\tau-1}=0$, the high types will become the dominant class, and if $n_{M}^{\tau-1}>0$, the pivotal vote will be in the hands of the medium class. We distinguish three cases.

Case iii1. Suppose that $n_{M}^{\tau-1}=0$ and $x_{M} \leq\left(X^{\tau-1}+x_{H}\right) / n^{\tau}$. 
By voting for a high type, the low types will lose power and they will get an egalitarian share today but a meritocratic one from tomorrow on. If they vote for a medium type, the pivotal vote tomorrow will be in the hands of the medium class. Given that $x_{M} \leq\left(X^{\tau-1}+x_{H}\right) / n^{\tau}$ by Case ii1 the medium type will keep the pivotal vote from there on and they will get egalitarianism forever. By Lemma 3 there exist $\bar{\delta}_{2}$ such that a low type prefers to hire a medium type rather than hiring a high type. And finally note that, hiring a low type is dominated by hiring a medium type because in both cases egalitarianism will be obtained, but the payoff by hiring a medium type is larger.

Case iii2. Suppose that $n_{M}^{\tau-1}=0$ and $\left(X^{\tau-1}+x_{H}\right) / n^{\tau} \leq x_{M}$.

In this case the low type will prefer to hire a medium type rather than a high type. In both cases the low types will lose political power. By hiring a high type they will have egalitarianism today and meritocracy forever. By hiring a medium type they will have egalitarianism today, meritocracy for some periods and then egalitarianism forever (because case ii 2 will apply). A similar argument to the one in Lemma 3 can be applied here to show that there exist $\bar{\delta}_{3}$ such that for all $\delta \geq \bar{\delta}_{3}$, the low type prefers to hire a medium type rather than a high type. So either the low type hires a medium type and loses political power or hires a low type and keeps it. In both cases egalitarianism will be, eventually, the sharing rule.

Case iii3. Suppose that $n_{M}^{\tau-1}>0$. In this case, by hiring a high type the low types will lose power in favor of the medium class which will keep the power forever if $\delta \geq \bar{\delta}_{M}$. Clearly this option dominates the option of hiring a medium type. Because by doing that they are also relinquishing power to the medium type, but the payoff today will be smaller. Finally, note that if $x_{M} \leq\left(X^{\tau-1}+x_{H}\right) / n^{\tau}$, egalitarianism will be the result from period $\tau$ on and this is better for the low type than hiring a new low type. If $x_{M}>\left(X^{\tau-1}+x_{H}\right) / n^{\tau}$ the low types will get egalitarianism today, meritocracy for some periods and, eventually egalitarianism again. If $\delta$ is sufficiently large, the loss in some periods due to meritocracy, will be compensated by a higher egalitarian payoff after those periods. If $\delta$ is not large enough, the low types will prefer to keep power. But also in this case the sharing rule will be meritocratic.

Summarizing, let $\bar{\delta}=\max \left\{\bar{\delta}_{M}, \bar{\delta}_{3}\right\}$. Then, for all $\delta \geq \bar{\delta}$, the proposition holds.

Proposition 1 says that when the future is very important, it is better for the high and the middle types to maintain political power that allows then to select the sharing rule and the hiring rather than hiring an agent with larger productivity that in the short run might increase production but may lead to losing the ability to select sharing rules and hirings in the future. In the case of the high type, they would only hire high types (like first class departments only hire high class scholars). Middle types may not be the most numerous, but they may be pivotal. And the lower class will yield power to the middle class because they know that the middle class will be egalitarian.

Three consequences of Proposition 1 are:

1. Exploitation, defined as the existence of an agent whose payment is less than her productivity, may arise in equilibrium in our democratic society in which voting -and not the possession of capital- is the deciding method.

2. Agents with high productivity may be left idle because their hiring might jeopardize the dominance of the ruling class. We propose to call this Political Unemployment. 
Of course, in order to have a complete theory of unemployment, we should also model the supply side and model how the market works. We have assumed that there is always a supply of all types in the market so the short side of the market -the organization- is the determinant of employment. This model is akin to the Marxist theory of the "reserve army" in which some workers are left idle in order to maintain low wages. In our case, high types are not hired because they constitute a threat to the power of the other classes.

3. The low class Relinquish Power to the middle class. The middle class is more productive and eventually will vote for egalitarianism so if they are hired, there is more surplus to share.

Proposition 1 has implications for the long term composition of the organization. Let $\left(m_{H}^{t}, m_{M}^{t}, m_{L}^{t}\right)$ be the proportion of insiders $H, M$ and $L$ respectively at time $t$. Let $\left(\tilde{m}_{M}, \tilde{m}_{M}, \tilde{m}_{L}\right)=\lim _{t \rightarrow \infty}\left(m_{H}^{t}, m_{M}^{t}, m_{L}^{t}\right)$.

Corollary 1 For a $\bar{\delta}$ sufficiently close to 1 if the dominant class at $t=0$ is $H$ then $\left(\tilde{m}_{M}, \tilde{m}_{M}, \tilde{m}_{L}\right)=(1,0,0)$. In any other case $\left(\tilde{m}_{M}, \tilde{m}_{M}, \tilde{m}_{L}\right)=(0.5,0.5,0)$.

The first part says that high quality organizations tend to continue with time. The examples of Cambridge, Harvard, La Sorbonne and other educational institutions that have been outstanding for a long time come to mind. ${ }^{2}$ The second part says that institutions started by another class of agents will never achieve full excellency. In this sense our model highlights the importance of the founding fathers of an organization.

We define the long run per capita output as $G=\lim _{t \rightarrow \infty}\left(m_{H}^{t} x_{H}+m_{M} x_{M}+m_{L}^{t} x_{L}\right)$. It is a measure of the efficiency of an organization. We now have the following:

Corollary 2 For a $\bar{\delta}$ sufficiently close to 1 if the dominant class at $t=0$ is $H$ then $G=x_{H}$. In any other case $G=0.5 x_{H}+0.5 x_{M}$.

We can define the instantaneous relative efficiency loss of $G$ as $\left(x_{H}-G\right) / x_{H}$. In any inefficient MPE this loss is $\left(x_{H}-0.5 x_{H}-0.5 x_{M}\right) / x_{H}=0.5\left(x_{H}-x_{M}\right) / x_{H}$. It ranges between zero and $50 \%$. Thus, any organization in which high types are not the dominant class at the start of the game, will have an inefficient mix of types. When, for instance, all the insiders in the first period are low types, the organization will experience a noteworthy increase in efficiency because in the long run high types will be half of the insiders. But it will never achieve full efficiency because this will imply that low or medium types will lose political power. This inefficiency is due to the fact that new hirings cannot sign a binding document in which they promise to vote for an egalitarian sharing rule. Thus, as pointed out by the Coase theorem, contractual incompleteness causes inefficiencies. A possible way to solve this would be to create two categories of agents: insiders at period zero who are the only ones with the right to vote (say they are tenured) and the rest. In this case, only high types will be hired and the organization will achieve almost full efficiency in the long run. Thus, from this perspective, the extension of voting rights to the newly hired creates inefficiencies.

\footnotetext{
2 But there are models of dynamic organizations in which this conclusion does not hold. In Sobel (2001) standards of admission and the average quality of incumbents rise or fall without any bound (which is impossible here because we only have three types). In Corchon (2005) there is free entry and an organization populated by high types may be subject to the entry of many low types whose life is easier under the command of a higher type. In our model there is no free entry.
} 


\section{Finitely living agents}

Our assumption that agents live an infinite number of periods is meant to free the model from the "last day effect". In this section we explore the consequences of assuming that agents live a finite number of periods. An agent in her last period inside the organization will be called retiring. In this new setting the state not only has to specify the number of each type of agents but who retires. We start with a simple observation: A retiring agent will always vote for hiring a high type. This is because junior members of the organization work but do not vote. In an egalitarian society it is a dominant strategy for a retiring agent to vote for the $H$ type regardless of what will happen tomorrow. If the society is meritocratic, then retiring agents are indifferent between any vote, but given A4, they will vote for a high type.

Since the results below are meant to highlight possibilities that may arise under finitely-living agents, we simplify the model here and we assume that there are only two types of agents. We also assume that, at most, one individual retires in each period. A state now includes the specification of the retiring agent.

We note first that, as in the previous section, if high types are the dominant class in some period, they will be the dominant class from there on.

Proposition 2 In any MPE if $H$ is the dominant class in period $\tau$, then $H$ is dominant in all subsequent periods and meritocracy will be the sharing rule.

Proof A type $H$ agent will always vote for a meritocratic sharing rule in any possible state. Given A4 and the remark above, a high type agent, independently of whether she is a retiring agent or not, will vote for hiring a high type. Thus, if $H$ is the dominant class at $\tau$, it will be the dominant class in any subsequent period.

Thus, the existence of retiring agents will never jeopardize the predominance of the $H$ type. However, this will not be the case for the low types. First note that, without retiring agents, an adaptation of Proposition 1 for two types will say that there exist $\bar{\delta}>0$ such that for all $\delta \geq \bar{\delta}$, if low types are the dominant class, they will be the dominant class forever and the sharing rule will be egalitarian. In the following proposition we show that with retiring agents, low types will lose political power in some subgames.

Proposition 3 Suppose that in $\tau, n_{H}^{\tau-1}+1=n_{L}^{\tau-1}$ and the retiring agent is a low type. Then, for all $\delta>0$, in any MPE low types will lose political power and meritocracy will be the sharing rule from $\tau+1$ on.

Proof In period $\tau$, the political power is in the hands of low types. Thus, egalitarianism will be the sharing rule. Given that high types and the retiring low type agents will vote for a high type, a high type will be hired and they will be the dominant class in the next period. By Proposition 2, high types will be the dominant class from then on and the resulting sharing rule will be meritocratic.

From Proposition 3 we learn that demographics have a lot to say and determine entirely that, in some organizations, power will change hands even if all agents are non myopic. 
Low types know that, in all subgames in which $n_{H}^{\tau-1}+1=n_{L}^{\tau-1}$ and the retiring agent is a low type, they will lose control. However, low types can be farsighted and maintain control by using the strategy described below:

Always vote for an egalitarian sharing rule. As a retiring agent, vote for a high type. As a non-retiring agent, if $n_{H}^{\tau-1}+2<n_{L}^{\tau-1}$ vote for a high type no matter who retires. If $n_{H}^{\tau-1}+2=n_{L}^{\tau-1}$ and the retiring agent is a high type, vote for a high type. If $n_{H}^{\tau-1}+2=n_{L}^{\tau-1}$ and the retiring agent is a low type, vote for a low type. And if $n_{H}^{\tau-1}+1 \geq n_{L}^{\tau-1}$ they vote for a high type.

The following proposition shows that $\bar{\sigma}=\left(\bar{\sigma}_{L}, \bar{\sigma}_{H}\right)$, where $\bar{\sigma}_{L}$ is the strategy described above and $\bar{\sigma}_{H}$ is such that in all periods high types vote for meritocracy and for hiring a high type, is a MPE for a sufficiently high $\delta$.

Proposition 4 There is $\bar{\delta}>0$ such that for all $\delta \geq \bar{\delta}, \bar{\sigma}=\left(\bar{\sigma}_{L}, \bar{\sigma}_{H}\right)$ is an MPE. Furthermore, if $n_{H}^{0}+1<n_{L}^{0}$, the lower class will be the dominant class forever.

Proof Note first that neither a high type, nor a retiring low type has incentives to deviate from their strategy. Note also that under this strategy, if low types are the dominant class, they will not lose political power and egalitarianism will be the sharing rule. In states where $n_{H}^{\tau-1}+2<n_{L}^{\tau-1}$, is a dominant strategy for the low type to vote for a high type. Independently of who the retiring agent is, low types will not lose political power and an egalitarian sharing rule gives larger payoffs with a high type. In states where $n_{H}^{\tau-1}+1 \geq n_{L}^{\tau-1}$, independently of who is retiring, a low type prefers to vote for a high type, either because they are not the dominant class and nothing will change with their vote (A4 applies here), or they are the dominant class. That is $n_{H}^{\tau-1}+1=n_{L}^{\tau-1}$, and the retiring agent is a high type (so hiring a high type will not change their political power), or $n_{H}^{\tau-1}+1=n_{L}^{\tau-1}$ and the retiring agent is a low type and therefore $n_{H}^{\tau-1}+1$ agents will vote for a high type (so a high type will be selected independently of the vote of the non-retiring low types). Finally, let us see that there are no profitable deviations in states where $n_{H}^{\tau-1}+2=n_{L}^{\tau-1}$. It is clear that no profitable deviation exists if the retiring agent is a high type. If the retiring agent is a low type and a non-retiring low type changes her vote and votes for a high type instead of a low type, a high type will be hired (a high type will receive the votes of the high types, the vote of the retiring low type and the vote of the deviant). Thus, this agent will receive a better payoff today (an egalitarian share with the surplus of a new high type), but the political power tomorrow will be in the hands of the high types and will stay in their hands forever. Let us see that there exist $\bar{\delta}$ such that this deviation is not profitable. By deviating, this agent will get

$$
\frac{X^{\tau-1}-x_{L}+x_{H}}{n^{\tau}}+\frac{\delta}{1-\delta} x_{L} .
$$

If she does not deviate from her strategy, in all subsequent periods $t \geq \tau, n_{H}^{t-1}+2=$ $n_{L}^{t-1}$. Furthermore, since a retiring low type (resp. high type) is replaced by a low type (resp. high type), $n^{t}=n^{\tau}$ for all $t \geq \tau$, and $X^{t-1}=X^{\tau-1}$. Then, the payoff associated with no deviation is: 


$$
\frac{X^{\tau-1}}{n^{\tau}}+\sum_{t>\tau} \delta^{t-\tau}\left(\frac{X^{\tau-1}}{n^{\tau}}\right)=\frac{1}{1-\delta}\left(\frac{X^{\tau-1}}{n^{\tau}}\right) .
$$

Thus, the deviation will not be profitable if

$$
\left(\frac{X^{\tau-1}}{n^{\tau}}\right) \geq(1-\delta) \frac{X^{\tau-1}-x_{L}+x_{H}}{n^{\tau}}+\delta x_{L} .
$$

Let $F(\delta)=(1-\delta)\left(X^{\tau-1}-x_{L}+x_{H}\right) / n^{\tau}+\delta x_{L}$. Note that $F(\delta)$ is decreasing in $\delta$, $F(0)>X^{\tau-1} / n^{\tau}$ and $F(1)<X^{\tau-1} / n^{\tau}$. Thus, there exist $\bar{\delta}$ such that for all $\delta \geq \bar{\delta}$, the deviation is not profitable.

The second part of the Proposition follows directly from the fact that $\bar{\sigma}$ is an MPE and starting from a dominant position, in the equilibrium path the low types will not lose political power.

\section{Conclusions and extensions of the model}

In this paper, we have studied the evolution of resource allocation in a society in which decisions are taken by plurality voting among three classes of citizens: highly $(H)$, medium $(M)$ and lowly $(L)$ productive. We have shown that if agents are sufficiently patient, if $H$ is dominant at time 0 , they will vote for meritocracy and they will hire $H$ types only. But in any other case, $M$ type agents become pivotal in the voting and, eventually, they will vote for egalitarianism and they will hire $M$ and $H$ agents in such a way that they stay pivotal. In particular, if $L$ agents are a majority at the starting time they will relinquish power to the $M$ class. As a result, some agents may be paid less than their productivity (exploitation) and highly productive agents may be left in the cold because their hiring will jeopardize the political power of the dominant class (political unemployment). In short, voting in the workplace does not avoid the shortcomings of capitalist economies. These shortcomings of voting -oppression of minorities or lack of efficiency- have been pointed out for a long time in the context of political systems.

The conclusions above were obtained in a particular model, but it seems to us that they are general. Dominant classes may relinquish power to more productive classes as long as they do not jeopardize output distribution. Thus, the Capitalist class relinquished power to the Managerial class for precisely the same reasons. Non dominant, but very productive classes, may be exploited by less productive classes who will also restrict the entry of potentially dangerous individuals into the organization. Finally, our results point out the importance of the initial constituency. A sometimes forgotten fact about the "Pilgrim Fathers" is their high level of education which might have pervaded the future of the US. William Brewster, John Harvard, Roger Williams, etc. were educated at Cambridge University. This initial constituency may help to explain why US converted easily from an agricultural economy into a high tech one, a conversion that was not so successful in other countries like Argentina and Australia (in both countries the initial constituency was very different from the US one). 
Our model can be extended in several directions. For example, the consideration of different tie breaking rules or a variable number of hirings will yield qualitatively identical predictions like our model. We mention below other possible extensions that require some reworking of our model.

1. More types.

In this case, if the political power is in the hands of one of the lower classes, they will relinquish it to the second most productive class. This class will hire highest productivity agents until they are almost in majority. And once the number of the highest productive agents is large enough, it will enforce an egalitarian distribution of the output.

2. Horizontal differentiation

In this case agents may have different characteristics -say, applied economists, macro and microeconomist. And each agent may prefer to have people from their own characteristics rather than people with other characteristics but larger productivity. In this case, political unemployment and exploitation may arise as a consequence of a single group becoming dominant in the voting and concentrating the hirings and the financing in its own group.

3. More general sharing rules

Voting on a large space of sharing rules is likely to produce no equilibrium as happens when voting on tax schedules. However, recently Brett and Weymark (2016) have shown that if agents can only propose tax schedules that are optimal for some agent and preferences are single-peaked the schedule proposed by the median skill is a Condorcet winner. It is likely that a similar result may hold in our model if agents vote on symmetric sharing rules. If this were the case, the equilibrium sharing rule would correspond to that preferred by the median productivity agents which, if the number of high types is large but not dominant, is the egalitarian.

4. Non Markovian strategies

A nagging question of our approach is what may happen if strategies are not required to be Markovian. In particular, we may conjecture if some kind of folk theorem may be true in our framework. A full blown investigation on the possibility of the folk theorem in our framework is beyond the scope of this paper. We just remark that the folk theorem is not true in general dynamic games and that the conditions found in the literature to obtain a folk theorem, are not met by our model. Thus Dutta (1995) requires that "for a folk theorem to hold, there must be some similarity in the possibilities from different states" (p. 10). This condition does not hold in our framework since the kind of punishments that you can apply by voting, is heavily dependent on the number of agents in each category, i.e. on the state. A recent contribution by Hörner et al. (2011) assumes that "the limit set of equilibrium payoffs is independent of the initial state" (p. 1281) which is not the case in our model in which the number of insiders in the first period determines long run payoffs.

Finally, there are other extensions that would require a complete revamping of our model and, therefore, despite the fact that they are important, are not pursued here: shortages of types in the market and competition between several organizations. In 
the first case voters have to balance carefully between hiring an agent which is not the first best today and waiting for a more suitable candidate in the future. This balance will depend on the difference in productivity between candidates and the waiting time and it is not likely to yield a clear-cut result. The second extension is to our taste the most promising. But it would require careful thought about how to model competition among organizations. We leave this for future research.

\section{Appendix}

First, let us introduce some notation. Let $V_{J}(T)$ be the continuation payoff for a type $J$ agent when hiring a type $T$ agent. Note first that $V_{L}(T) \leq V_{M}(T) \leq V_{H}(T)$. This is because in each period, the rule is either meritocratic or egalitarian. If it is egalitarian, everyone receives the same, if it is meritocratic the higher the type the higher the payoff obtained.

Proof of Lemma 1 Given A5, a high type agent will always vote for a meritocratic sharing rule and a low type agent for an egalitarian rule independently of the hiring decision. Thus, when there is no dominant class, when deciding on the sharing rule, the pivotal voter is a medium type agent. Let us see that for $\delta$ sufficiently high, the medium type is also pivotal when voting on the hiring. First note that, under A4 and A5, an agent will always vote for hiring another agent oh her type or of a higher type. Thus, if a medium type prefers to hire a high type, a high type will be hired. Furthermore, given $\mathrm{A} 4$, and $\mathrm{A} 5$, in all $\tau$ such that $n_{H}^{\tau-1}+1>n_{M}^{\tau-1}+n_{L}^{\tau-1}$, a medium type will always prefer to hire a high type because her pivotal vote on the sharing rule for the next period is not at risk. If $n_{H}^{\tau-1}+1=n_{M}^{\tau-1}+n_{L}^{\tau-1}$, there is a trade off because by hiring a high type, the medium class will lose its pivotal vote on the sharing rule in the next period in favor of the high type, and by part (i) in Proposition 1 we will get meritocracy from there on. To discuss the implications of the voting behavior we distinguish three cases.

(i) Suppose that at $\tau,\left(X^{\tau-1}+x_{H}\right) / n^{\tau} \leq x_{M}$.

Since the pivotal voter on the sharing rule is the medium type, she will vote for a meritocratic sharing rule independently of the new hiring. Suppose that the medium type prefers to hire another medium type. Thus,

$$
x_{M}+\delta V_{M}(M)>x_{M}+\delta V_{M}(H)=x_{M}+\frac{\delta}{1-\delta} x_{M}
$$

Which implies that some of the terms in $V_{M}(M)$ are the result of an egalitarian rule with a payoff greater than $x_{M}$. Given that $V_{L}(T) \geq(1 /(1-\delta)) x_{L}$ for all $T \in\{H, M, L\}$, $V_{L}(M)>(1 /(1-\delta)) x_{L}$. Thus, in this situation a low type agent will also prefer to hire a medium type. As a result, a medium type will be hired.

(ii) Suppose that at $\tau, x_{M} \leq X^{\tau-1} / n^{\tau-1}<\left(X^{\tau-1}+x_{H}\right) / n^{\tau}$.

Since the pivotal voter on the sharing rule is the medium type, she will vote for an egalitarian sharing rule independently of hiring a high or a medium type. Suppose that 
the medium type prefers to hire another medium type. Thus,

$$
\frac{X^{\tau-1}+x_{M}}{n^{\tau}}+\delta V_{M}(M)>\frac{X^{\tau-1}+x_{H}}{n^{\tau}}+\frac{\delta}{1-\delta} x_{M} .
$$

Or equivalently

$$
\frac{X^{\tau-1}+x_{H}}{n^{\tau}}-\frac{X^{\tau-1}+x_{M}}{n^{\tau}}<\delta\left(V_{M}(M)-\frac{1}{1-\delta} x_{M}\right)
$$

Let us see that the low type also prefers to vote for a medium type. Note that $V_{M}(M)$ and $V_{L}(M)$ only differ in the periods where meritocracy is the resulting sharing rule (if any of these periods exist). Thus,

$$
V_{M}(M)-V_{L}(M)<\frac{1}{1-\delta}\left(x_{M}-x_{L}\right)
$$

Therefore, if (6.3) holds, then

$$
\frac{X^{\tau-1}+x_{H}}{n^{\tau}}-\frac{X^{\tau-1}+x_{M}}{n^{\tau}}<\delta\left(V_{L}(M)-\frac{1}{1-\delta} x_{L}\right)
$$

also holds and the low type will also prefer to hire a medium type.

(iii) Suppose that at $\tau, X^{\tau-1} / n^{\tau-1} \leq x_{M}<\left(X^{\tau-1}+x_{H}\right) / n^{\tau}$.

Since the pivotal voter on the sharing rule is the medium type, she will vote for a meritocratic sharing rule if a medium type is hired, and for an egalitarian one if a high type is hired. Suppose that the medium type prefers to hire another medium type. Thus,

$$
x_{M}+\delta V_{M}(M)>\frac{X^{\tau-1}+x_{H}}{n^{\tau}}+\frac{\delta}{1-\delta} x_{M} .
$$

Or equivalently

$$
\frac{1-2 \delta}{1-\delta} x_{M}+\delta V_{M}(M)>\frac{X^{\tau-1}+x_{H}}{n^{\tau}} .
$$

Because in all periods where the pivotal vote of the medium type on the sharing rule is not at risk a new high type is hired, from $\tau+1$ on, the sharing rule will be egalitarian. This is because once we hire a high type, the average surplus is above $x_{M}$ and from there on, regardless of whether a high or a medium type is hired, the average surplus will remain above $x_{M}$. Thus, $V_{M}(M)=V_{L}(M)$. Furthermore, let us see that the left hand side of (6.7) is increasing in $\delta$. Let $y_{t}$ denote the t-term in $V_{M}(M)$, recall that $y_{t}>x_{M}$ for all $t$. The derivative with respect to $\delta$ of the left hand side of (6.7) is

$$
\frac{-1}{(1-\delta)^{2}} x_{M}+V_{M}(M)+\delta \frac{\partial V_{M}(M)}{\partial \delta}
$$


Given that $V_{M}(M)=\sum_{t=0}^{\infty} \delta^{t} y_{t}>\sum_{t=0}^{\infty} \delta^{t} x_{M}=(1 /(1-\delta)) x_{M}$, and

$$
\frac{\partial V_{M}(M)}{\partial \delta}=\sum_{t=1}^{\infty} t \delta^{t-1} y_{t}>\sum_{t=1}^{\infty} t \delta^{t-1} x_{M}=\left(1 /(1-\delta)^{2}\right) x_{M},
$$

then (6.8) is positive. Thus, the left hand side of (6.7) is increasing in $\delta$. Therefore, if (6.7) holds for some $\delta$, it holds for all $\delta^{\prime}>\delta$. Finally note that for $\delta>0.5$, whenever (6.7) holds,

$$
\frac{1-2 \delta}{1-\delta} x_{L}+\delta V_{L}(M)>\frac{X^{\tau-1}+x_{H}}{n^{\tau}}
$$

also holds and thus low types will also prefer to hire a medium type.

Summarizing, there exist $\bar{\delta}_{0}>0.5$ such that for all $\delta \geq \bar{\delta}_{0}$ in any MPE, if at period $\tau$ there is no a dominant class, a type $M$ agent is the pivotal voter.

Proof of Lemma 2 First of all note that $\delta V_{E}^{1}(\delta)$ is

$$
\begin{aligned}
\delta V_{E}^{1}(\delta)= & \sum_{t=0}^{\infty} \delta^{2 t+1}\left[\frac{X^{\tau-1}+(t+1) x_{M}+(t+1) x_{H}}{n^{\tau}+2 t+1}\right] \\
& +\sum_{t=1}^{\infty} \delta^{2 t}\left[\frac{X^{\tau-1}+(t+1) x_{M}+t x_{H}}{n^{\tau}+2 t}\right]
\end{aligned}
$$

The first term corresponds to all the periods where a high type is hired and the second term to the periods where a medium type is hired. First, note that since $\delta<1$, all power series in $\delta V_{E}^{1}(\delta)$ are convergent. Note also that all terms in $\delta V_{E}^{1}(\delta)$ are larger than $x_{M}$. Let us see that there exist $\bar{\delta}_{1}>0.5$ such that the first three terms in $x_{M}+\delta V_{E}^{1}(\delta)$ are bigger than $\left(X^{\tau-1}+x_{H}\right) / n^{\tau}+\delta x_{M}+\delta^{2} x_{M}$. Formally, let us see that there is $\bar{\delta}_{1}>0.5$ such that for all $\delta>\bar{\delta}_{1}$,

$$
\begin{gathered}
x_{M}+\delta\left(\frac{X^{\tau-1}+x_{M}+x_{H}}{n^{\tau}+1}\right)+\delta^{2}\left(\frac{X^{\tau-1}+2 x_{M}+x_{H}}{n^{\tau}+2}\right) \\
>\frac{X^{\tau-1}+x_{H}}{n^{\tau}}+\delta x_{M}+\delta^{2} x_{M} .
\end{gathered}
$$

For $\delta=0$, the left hand side of (6.12) is $x_{M}$ which is smaller than $\left(X^{\tau-1}+x_{H}\right) / n^{\tau}$. As $\delta$ converges to 1 , the left hand side converges to

$$
x_{M}+\frac{X^{\tau-1}+x_{M}+x_{H}}{n^{\tau}+1}+\frac{X^{\tau-1}+2 x_{M}+x_{H}}{n^{\tau}+2},
$$

and the right hand side converges to

$$
\frac{X^{\tau-1}+x_{H}}{n^{\tau}}+x_{M}+x_{M}
$$


Let us see that

$$
\frac{X^{\tau-1}+x_{M}+x_{H}}{n^{\tau}+1}+\frac{X^{\tau-1}+2 x_{M}+x_{H}}{n^{\tau}+2}>\frac{X^{\tau-1}+x_{H}}{n^{\tau}}+x_{M},
$$

or equivalently,

$$
\begin{aligned}
\frac{X^{\tau-1}+2 x_{M}+x_{H}}{n^{\tau}+2}-x_{M} & >\frac{X^{\tau-1}+x_{H}}{n^{\tau}}-\frac{X^{\tau-1}+x_{M}+x_{H}}{n^{\tau}+1}, \\
\frac{X^{\tau-1}+x_{H}-n^{\tau} x_{M}}{n^{\tau}+2} & >\frac{X^{\tau-1}+x_{H}-n^{\tau} x_{M}}{n^{\tau}\left(n^{\tau}+1\right)}
\end{aligned}
$$

Since $n^{\tau} \geq 2, n^{\tau}\left(n^{\tau}+1\right)$ is larger than $\left(n^{\tau}+2\right),(6.15)$ holds.

Let

$$
F(\delta)=\left(1-\delta-\delta^{2}\right) x_{M}+\delta\left(\frac{X^{\tau-1}+x_{M}+x_{H}}{n^{\tau}+1}\right)+\delta^{2}\left(\frac{X^{\tau-1}+2 x_{M}+x_{H}}{n^{\tau}+2}\right) .
$$

We have seen that $F(0)<\left(X^{\tau-1}+x_{H}\right) / n^{\tau}$, and that $\lim _{\delta \rightarrow 1} F(\delta)>\left(X^{\tau-1}+x_{H}\right) / n^{\tau}$, note also that since $\left(X^{\tau-1}+x_{M}+x_{H}\right) /\left(n^{\tau}+1\right)>x_{M}$, and $\left(X^{\tau-1}+2 x_{M}+x_{H}\right) /\left(n^{\tau}+\right.$ 2) $>x_{M}, F(\delta)$ is increasing in $\delta$. Thus, we can find $\bar{\delta}_{1}>0.5$ such that for all $\delta>\bar{\delta}_{1}$, $F(\delta)>\left(X^{\tau-1}+x_{H}\right) / n^{\tau}$, as we wanted to prove.

Finally, note that for $\delta>0.5$, if (3.1) holds, then (3.2) also holds.

Proof of Lemma 3 Note first that if (3.3) holds, given that $x_{M}>x_{L}$, (3.4) also holds. Also note that $\delta V_{E}^{2}(\delta)$ can be written as (6.11) in Lemma 2. As in Lemma 2, all terms in $\delta V_{E}^{2}(\delta)$ are larger than $x_{M}$. Let us see that there exist $\bar{\delta}_{2}$ such that the first three terms in $\left(X^{\tau-1}+x_{M}\right) / n^{\tau}+\delta V_{E}^{2}(\delta)$ are larger than $\left(X^{\tau-1}+x_{H}\right) / n^{\tau}+\delta x_{M}+\delta^{2} x_{M}$. That is, there is $\bar{\delta}_{2}$ such that in three periods, we compensate the initial loss of getting $\left(X^{\tau-1}+x_{M}\right) / n^{\tau}$ compared with obtaining $\left(X^{\tau-1}+x_{H}\right) / n^{\tau}$. Formally, let us see that there is $\bar{\delta}_{2}$ such that for all $\delta>\bar{\delta}_{2}$,

$$
\begin{aligned}
\left(X^{\tau-1}+x_{M}\right) / n^{\tau} & +\delta\left(\frac{X^{\tau-1}+x_{M}+x_{H}}{n^{\tau}+1}\right) \\
& +\delta^{2}\left(\frac{X^{\tau-1}+2 x_{M}+x_{H}}{n^{\tau}+2}\right)>\frac{X^{\tau-1}+x_{H}}{n^{\tau}}+\delta x_{M}+\delta^{2} x_{M} .
\end{aligned}
$$

Given that $\left(X^{\tau-1}+x_{M}\right) / n^{\tau}>x_{M}$, we can apply the argument in Lemma 2 to ensure the existence of $\bar{\delta}_{2}$.

\section{References}

Acemoglu D, Robinson JA (2000) Why did the west extend the franchise? democracy, inequality, and growth in historical perspective. Q J Econ 115(4):1167-1199

Barberá S, Maschler M, Shalev J (2001) Voting for voters: a model of electoral evolution. Games Econ Behav 37:40-78 
Barberá S, Beviá C, Ponsatí C (2015) Meritocracy, egalitarianism and the stability of majoritarian organizations. Games Econ Behav 91:237-257

Brett C, Weymark J A (2016) Voting over selfishly optimal nonlinear income tax schedules with a minimum-utility constraint. Vanderbilt University Department of Economics Working Papers 1600005, Vanderbilt University Department of Economics

Conley JP, Temini A (2001) Endogenous enfranchisment when groups' preferences conflict. J Polit Econ 109(1):79-102

Corchon LC (2005) Monk business: an example of the dynamics of organizations. Soc Choice Welf 24:543556

Duffie D, Geanakoplos J, Mas-Colell A, McLennan A (1994) Stationary Markov equilibria. Econometrica 62(4):745-781

Duggan J, Martinelli C (2015) The political economy of dynamic elections: a survey and some new results. Mimeo http://www.johnduggan.net/

Dutta PK (1995) A folk theorem for stochastic games. J Econ Theory 66:1-32

Dziuda W, Loeper A (2015) Dynamic collective choice with endogenous status quo. J Polit Econ (Forthcoming)

Guesnerie R, Woodford M (1992) Endogenous fluctuations. In: Laffont J-J (ed) Advances in economic theory, vol II. Cambridge University Press, Cambridge

Hörner J, Sugaya T, Takahashi S, Vieille N (2011) Recursive methods in discounted stochastic games: an algorithm for $\delta \rightarrow 1$ and a folk theorem. Econometrica 79(4):1277-1318

Jack W, Lagunoff R (2006) Dynmic enfranchisement. J Publ Econ 90:551-572

Kang S (1988) Fair distribution rule in a cooperative enterprise. J Comp Econ 12(1):89-92

Lizzeri A, Persico N (2004) Why did the elites extend the suffrage?. Q J Econ May 119(2):707-765

Llavador H, Oxoby RJ (2005) Partisan competition, growth and the franchise. Q J Econ 120(3):1155-1189

Mavridis C, Serena M (2015) Costly voting under complete information. Mimeo, Universidad Carlos III de Madrid

Razin A, Sadka E, Suwankiri B (2016) The welfare State and migration: a dynamic analysis of political coalitions. Res Econ 70(1):122-142

Roberts K (2015) Dynamic voting in clubs. Res Econ 69(3):320-335

Roemer J (1982a) A general theory of exploitation and class. Harvard University Press, Cambridge

Roemer J (1982b) Property relations versus surplus value in marxian exploitation. Phil Publ Aff 11(4):281313

Roemer J (1982c) Methodological individuals and analytical marxism. Theory Soc 11:513-520

Roemer J (1985) Should marxist be interested in exploitation? Phil Publ Aff 14:30-65

Roemer J (1988) Free to lose. Harvard University Press, Cambridge

Sobel J (2001) On the dynamics of standards. RAND J Econ 32(4):606-623

Zapal J (2014) Simple Markovian equilibria in dynamic spatial legislative bargaining. CERGE-EI Working Paper Series No. 515 